\title{
Percutaneous Drug Penetration: Choosing Candidates for Transdermal Development
}

\author{
Gordon L. Flynn and Barbra Stewart \\ College of Pharmacy, Pharmaceutics Group, College of Pharmacy, \\ The University of Michigan, Ann Arbor
}

\begin{abstract}
Flynn, G.L., and B. Stewart: Percutaneous drug penetration: Choosing candidates for transdermal drug development. Drug Dev. Res. 13:169-185, 1988.

There is currently a high level of interest in using the skin as a route for delivering drugs. One hears the questions: What are the attributes of a drug that make it a serious candidate for transdermal delivery? By what a priori analysis might one zero in on the best transdermal candidate within a family of drugs? Answers to these questions lie in understanding the molecular factors that make a drug a facile permeant of the skin. Among other properties, it must have a high absolute affinity for the skin's phases, which provide for its diffusive conduction. Other factors in evaluation are the potency of the drug and the relative efficiency of the drug's systemic presentation once it has gained access to the body. One also considers the potential for the drug to elicit adverse responses in the skin. Fortunately, parallels between the drug's ability to partition between oil and water and its ease of mass transfer across the skin can be used to ferret out a working mass transfer coefficient. If not already known, solubilities are easily experimentally deduced. The extent of first-pass metabolism by the oral route, presumed to be a known quantity, is compared with the relative amount of metabolism of the drug in the course of its diffusion through the skin, an experimentally determined quantity, in order to set the transdermal dose. These bits of information can then be used to form an early, reasonably faithful picture of the feasibility of delivering a particular drug transdermally and to make a first estimate of the size of patch required for the drug.
\end{abstract}

Key words: skin permeability, transdermal delivery, percutaneous absorption, structureactivity relationships, selecting drug candidates

Received final version January 26, 1988; accepted Janaury 28, 1988.

Address reprint requests to Dr. Gordon L. Flynn, College of Pharmacy, The University of Michigan, Ann Arbor, Ml 48109-1065.

(C) 1988 Alan R. Liss, Inc. 


\section{INTRODUCTION}

For as long as it has been appreciated that substances exist in nature that alleviate pain and suffering, man has sought the means to administer them to gain their full advantage. One can presume that the oral route figured prominently in prehistoric medicine, for taking drugs orally is as natural as eating. One can also presume that early man encountered some of the general difficulties that still exist with the oral route, namely problems attending administration of drugs to nauseous and unconscious patients. Alternative methods of administration were surely attempted, with the topical route certainly amongst those tried, given its accessibility and extensiveness. Drugs were apparently used topically by early Egyptian and other Mediterranean civilizations not only to treat surface wounds and the local manifestations of disease but also to cure general illnesses. Poultices, cataplasms, and plasters were still in wide use as cold remedies and for other human afflictions at the dawn of the 20th century. However, only in the last 20 years has it proven possible to unequivocally demonstrate that systemic therapy by the topical route, though subject to severe constraints, is indeed feasible.

We seem to be passing through a period in which the commercial lure of transdermal delivery is running out in front of our comprehension of the administration method. Many, many hours have therefore been spent in the laboratory and clinic learning the hard way that many drugs fail stringent tests of potency and toxicity requisite for their delivery through the skin. Wouldn't it be nice if screening to sort out the most promising drugs for the method could be accomplished in the library with the aid of pencil and paper and a keen sense of the processes involved? If one can accept an order of magnitude answer, this is, in fact, possible! The present report aims to establish this point. It catalogs obstacles to the successful deployment of the transdermal method of delivery and especially concentrates on the physicochemical and biophysical constraints associated with permeation of the skin. The relatively impermeable strata of skin interposed between the surface of the body and the systemic circulation are a prime consideration. Although a conservative stance is taken with respect to the delivery method, this report actually places transdermal delivery in a positive light, for, when the principles set forth are applied, one can rule out unworthy drugs and concentrate effort on candidates of high promise. It emphasizes two points: 1) the information required for the initial judgement about the transdermal potentials of drugs; and 2) the application of the information to make enlightened decisions. While the topical delivery of drugs to achieve their systemic effects is of sole concern, the general approach to the selection of drug candidates can also be applied to the selection of drugs best suited for local therapy.

\section{PERCUTANEOUS ABSORPTION}

\section{Transepidermal Absorption}

Percutaneous absorption involves passive diffusion of substances through the skin. The mechanism of permeation can involve passage through the epidermis itself or diffusion through shunts, particularly those offered by the relatively ubiquitously distributed hair follicles and eccrine glands (see Fig. 1). While exceptions to the rule are acknowledged, it is now generally believed that the transepidermal pathway is principally responsible for diffusion across the skin. Far more often than not, the main resistance encountered along this pathway arises in the stratum corneum. Characterization of the physical nature of the skin barrier thus should begin with characterization of the stratum corneum.

Over most of the body, the stratum corneum is composed of 15 to 25 layers of "acutely flattened, metabolically inactive cells" [Odland, 1983]. It is surprisingly dense for a soft tissue, having an estimated dry weight density of $1.3-1.4 \mathrm{gm} / \mathrm{cm}^{3}$ [Scheuplein, 1978; Scheuplein and Bronaugh, 1983]. The thickness of individual cell layers varies, ranging from $0.2 \mu \mathrm{m}$ to $0.5 \mu \mathrm{m}$, depending on location [Plewig et al., 1983], but appears to be uniform at a location. The individual platelets, which function as building blocks, appear as crudely 


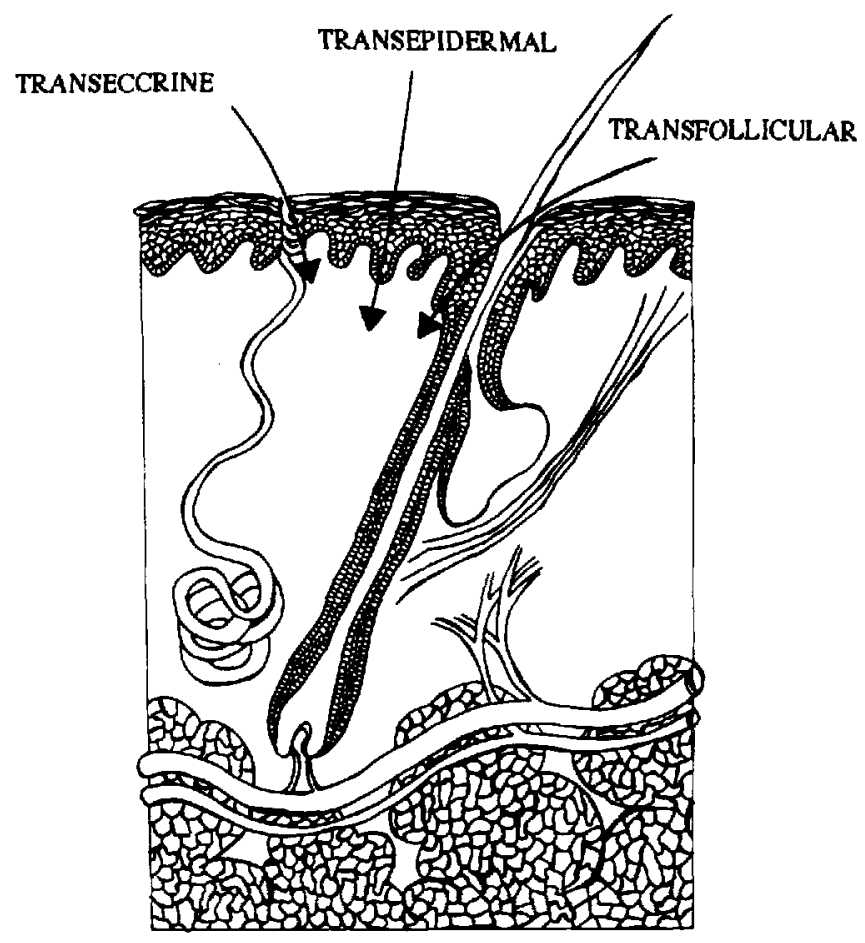

Fig. 1. Illustration showing the possible routes for percutaneous absorption. Currently it is believed that a transepidermal route through the seams between the "cells" of the stratum corneum is the principal pathway for most drugs.

shaped polygons. In their longest dimension these measure $30-40 \mu \mathrm{m}$. By the time the cell platelets are laid into the stratum corneum, the cell interior is crisscrossed with densely packed bundles of keratin fibers [Steinert, 1983]. Due to this, the dry composition of the horny layer is $75-85 \%$ protein, most of which is intracellular keratin. A fraction of the protein also appears to be associated with a still intact network of cell membranes. The bulk of the remainder of the substance of the stratum corneum is lipids. Elias and coworkers [1983] make a strong case that the stratum corneum's lipoidal substance lies almost exclusively in the seams between cells. It appears to be organized into bilayers. The greater part of this lipid originates from membrane coating granules found in the granular layer. These pass from the granular cell's interior into the intercellular space as one of the culminating events of differentiation of the epidermal keratinocyte. The stratum corneum thus has two distinct chemical regimes, the mass of intracellular protein and the intercellular lipoidal medium. These phases are isolated from one another by still present, and presumably intact, cell membranes. The cell membranes themselves are knit together by desmosomes, adding a tough infrastructure to the horny mass [Goldsmith, 1983]. This conceived structural organization supports the idea that a lipoidal route exists between the cells, possibly with alternative pathways through the mass of protein [Scheuplein, 1965, 1967, 1976, 1978; Scheuplein and Bronaugh, 1983; Katz and Poulsen, 1971; Flynn, 1985]. The intercellular lipid route occupies $1 \%$ of the stratum corneum's productive diffusional area (area in the plane of the skin and perpendicular to permeation) by rough estimate [Flynn, 1985]. As meager as it is, this description captures most of what is accepted about the organization of the stratum corneum's physically distinct phases. The picture arrived at is to an uncomfortable degree still tentative, making a more refined description of the tissue's potential diffusion pathways hazardous. 
When a permeating drug exits the stratum corneum, it enters the wet cell mass of the epidermis, and, since the epidermis has no direct blood supply, the drug is forced to diffuse across it to reach the vasculature immediately beneath. Given that this mass of viable cells gives rise to the stratum corneum, it is remarkable how different its barrier properties are. In the live epidermis several histologically distinguishable layers of closely packed cells form a tight, cellular continuum $50-100 \mu \mathrm{m}$ in thickness. At its base, the epidermis forms an irregular, papillate interface with the dermis. The basal cells are overlaid on a thin basement membrane and are anchored to the dermis by tiny fibers. Proliferating stem cells in the basal layer give rise to all epidermal cell layers above. The basement cells tend to be cuboidal or columnar in shape, but, as they progress toward the horny layer, they undergo profound changes in appearance. Sharp, spiny protuberances form at points of cell attachment immediately above the basal layer (stratum spinosum). As the cells near the undersurface of the stratum corneum, they flatten considerably and stain to give a granular appearance (stratum granulosum). The nucleus and cell organelles are still visible in the granular cell's interior as are keratohyalin substance, the preferential staining of which gives the granular appearance, and small lipid-filled vesicles known as membrane coating granules. The latter particles pass by exocytosis into the space between cells in the final stage of formation of the stratum corneum. Though appearing granular and full of vesicles and cell organelles, there is little reason to believe that the barrier qualities of the granular layer are unique within the viable epidermis. Rather, and as a practical matter, the viable epidermis, mitotic layer to granular layer, is considered as a single field of diffusion in models. A considerable amount of experimental and other evidence indicates it is a permeable field that functions as a viscid watery regime to most permeants [Scheuplein, 1965, 1967, 1976; Flynn, 1985]. It appears that only ions and polar nonelectrolytes found at the hydrophilic extreme and lipophilic nonelectrolytes at the hydrophobic extreme have any real difficulty passing through the viable field [Flynn, 1985]. The epidermal cell membranes are tightly joined and there is little to no intercellular space for ions and polar nonelectrolyte molecules to diffusionally squeeze through. Thus permeation must follow a course that requires frequent crossings of cell membranes, each crossing being a thermodynamically prohibitive event for such water-soluble species. Extremely lipophilic molecules, on the other hand, are thermodynamically constrained from dissolving in the watery regime of the cell (the cell cytoplasm).

A rich bed of capillaries is encountered $20 \mu \mathrm{m}$ or so into the dermal field. Passage through this dermal region represents a final hurdle to systemic entry. This is so regardless of whether permeation is transepidermal or by a shunt route. The dermal construction is a mesh of collagen fibers and is the principal structural element of skin. Wide spaces between the bundles of collagen fibers are filled with a watery gel called the ground substance. Permeation through the dermis is through the interlocking channels of ground substance formed between fibers. The gaps between fibers are far too wide to filter large molecules and thus, as far as can be told from existing information, diffusion through the dermis is facile and without molecular selectivity. Because the viable epidermis and dermis lack major physicochemical distinction, they are generally considered as a single field of diffusion [Flynn, 1985]. An exception to this generalization is made for solutes of extreme polarity, as the epidermis offers a factorable resistance to such species.

Permeation by the transepidermal route first involves partitioning into the stratum corneum. Diffusion across this tissue, the viable epidermis, and the upper dermis then ensues. The current popular belief is that most substances diffuse across the stratum corneum via a lipoidal pathway, which recent histological evidence places between the cells [Elias et al., 1983]. This is a tortuous pathway of limited fractional volume and even more limited productive fractional area in the plane of diffusion. The presence and nature of this pathway explain the greater part of the existing chemical structure-skin permeation data [Flynn, 1985]. However, there appears to be another microscopic path through the stratum corneum for extremely polar compounds and ions. Otherwise these would not permeate at rates that are 
measurable, considering their $\mathrm{o} / \mathrm{w}$ distributioning tendencies [Flynn, 1985]. In the other extreme of polarity, lipophilic molecules concentrate in and diffuse with relative facility through the horny layer's intercellular regime only to find themselves blocked by the wet cell mass beneath. Thus the viable tissue is rate-determining when nonpolar compounds are involved [Scheuplein, 1965, 1967, 1976; Flynn, 1985].

\section{Transfollicular (Shunt Pathway) Absorption}

Arguments have raged about the true pathway for percutaneous absorption, but it is now the generally accepted view that the skin's appendages offer only secondary avenues for the permeation of most chemicals. Since pilosebaceous and eccrine glands are distributed over the entire body, except for some very limited, isolated areas, only these appendages are ever taken seriously as shunts bypassing the stratum corneum. Though eccrine glands are numerous, up to 400 glands per $\mathrm{cm}^{2}$ in places such as the palms and soles, their orifices are tiny and add up to a miniscule fraction of the body's surface. Moreover, they are either evacuated or so profusely active that it is unlikely that molecules can diffuse inwardly against the gland's output. For these reasons they are discounted as a significant route for percutaneous absorption.

Pilosebaceous glands are less numerous than eccrine glands, having a density variously estimated to be between 50 and 100 glands per $\mathrm{cm}^{2}$ of body surface. However, the opening of the follicular pore where the hair shaft exits the skin is relatively large; the fractional area of the route has been estimated to be as high as 1/1,000th of the skin's surface [Flynn, 1985]. Moreover, the duct of the gland is filled with a soft, slowly extruded lipoidal medium, sebum, in which lipophilic drugs should be soluble and through which they should diffuse with ease. Thus the follicular route remains as a possibly important avenue for percutaneous absorption. Partitioning into sebum followed by diffusion through the sebum to the depths of the epidermis is the envisioned mechanism of permeation by this route. Vasculature subserving the hair follicle located in the dermis represents the likely point of systemic entry.

\section{Clearance by the Local Circulation}

The earliest possible point of entry of drugs and chemicals into the systemic circulation is within the papillary plexus in the upper dermis. The process of percutaneous absorption is generally regarded as ending at this point, a presumption that is almost certainly a model-based oversimplification, as some molecules clearly bypass the circulation and diffuse deeper into the dermis. Blood flow through the superficial capillary plexus is highly variable. For one thing, the level of perfusion of blood is determined by the existing physiological need to conserve or eliminate body heat. Whether the flow of blood through chilled skin is adequate for efficient local clearance of permeant is an important question having no current answer. Blood flow is also exaggeratedly increased by local trauma. On the other hand, clearance may be diminished in old age due to vascular decrepitude. This thought has sparked the suggestion that aged skin is more prone to the allergic and irritant effects of topically contacted compounds [Kligman, 1979]. Despite these collective concerns, the preponderance of evidence seems to indicate that local clearance is relatively efficient.

The above is a thumbnail sketch of the principal features of the skin as they relate to the inward diffusion of chemicals from the skin's surface. It is clear that the development of models for skin permeation must include diffusion across a series of barriers and possibly diffusion through parallel pathways. Moreover, certain anatomical structures of the skin are relatively impervious, a factor that limits the available area for diffusion and forces permeation mainly through the limited fraction of soft lipoidal material between the stratum corneum cells or through sebum in the follicular duct. Clearance is by way of the blood, the flow of which varies depending on momentary physiological need. 


\section{SKIN PERMEATION ASSESSMENT Diffusion From a Saturated Medium in Contact With the Skin}

In order to make an assessment of the systemic availability of a topically applied drug (or topically contacted chemical), one normally has to consider the separate but concerted delivery processes of drug release from the topical vehicle (or medium containing the chemical) into the skin and diffusion through the skin. However, to assess whether a drug can be delivered transdermally for systemic therapy, it is only necessary to consider the latter process. This is so because it is presumed ab initio that the means will be found to maximize (or optimize) a drug's thermodynamic potential at the skin's surface, providing the drug's percutaneous absorption rate is consistent with therapeutic needs. Removing all considerations of vehicle control to make the first assessment of feasibility of delivery considerably simplifies the analysis. What one in effect does is estimate the maximum rate at which the drug can be caused to diffuse across the skin. It's easy to back off from this rate should it be overly fast. The maximum rate will be achieved at the attainable limit of thermodynamic activity of the drug. For a crystalline drug (most drugs of interest are going to be in the form of molecular crystals), the maximum attainable thermodynamic activity is set by the neat solid. Therefore, for the purpose of establishing feasibility, transdermal drug delivery from a continuously saturated solution through the skin is assumed. While the dominant resistance of the skin usually lies in its stratum corneum, the outermost, 10 - $\mu \mathrm{m}$-thick, devitalized layer of the tissue, there are times and circumstances when other strata of the skin in series with the stratum corneum offer appreciable resistances to diffusion or when shunt routes are followed and cognizance is taken of this in the general analysis.

Situations in which a drug is delivered from a reservoir in which it is at unit activity (from a saturated solution) through a resistant membrane like the stratum corneum (or whole skin) are frequently encountered in transdermal delivery. Equation 1 mathematically describes the delivery dependency:

$$
M_{t}=\left(K \cdot C_{s}\right) \cdot\left(\frac{D_{s c} \cdot t}{h_{s c}}-\frac{h_{s c}}{6}-\frac{2 \cdot h_{s c}}{\pi^{2}} \cdot \sum_{i=1}^{x} \frac{(-1)^{n}}{n^{2}} \cdot e\left(\frac{-D_{s c} \cdot n^{2} \cdot \pi^{2} \cdot t}{h_{s c}^{2}}\right)\right)
$$

Here $\mathrm{M}_{\mathrm{t}}$ is the amount of a drug passing through a unit area of the membrane in time, $t$, under conditions that the drug is delivered from a saturated reservoir of concentration, $\mathrm{C}_{\mathrm{s}} . \mathrm{K}$ is the skin/vehicle partition coefficient, making the product, $\mathrm{K} \cdot \mathrm{C}_{\mathrm{s}}$, the saturation concentration of the drug within the skin's surface. The terms within the summation, $D_{\mathrm{sc}}$ and $\mathrm{h}_{\mathrm{sc}}$, are the effective diffusion coefficient and the effective thickness of the stratum corneum or, more generally, the membrane, respectively. Though subscripted with sc to designate the stratum corneum, they are in fact functional parameters representing the net properties of all consequential strata. Even when the stratum corneum is the sole source of diffusional resistance, $D_{s c}$ and $h_{s c}$ are effective parameters because there is no way to make an explicit accounting for important factors such as the fractional area and tortuosity of the route taken through the stratum corneum.

The theoretical curve (schematically drawn) depicted in Figure 2 is based on equation 1. The curve indicates that no drug or very little drug penetrates the skin in the early moments after the application of the drug-containing reservoir. However, once the first molecules breach the membrane, the flux of the drug gradually grows until it reaches a steady level. One obtains the equation for the steady-state line by allowing $t \rightarrow \infty$, which leads to: 


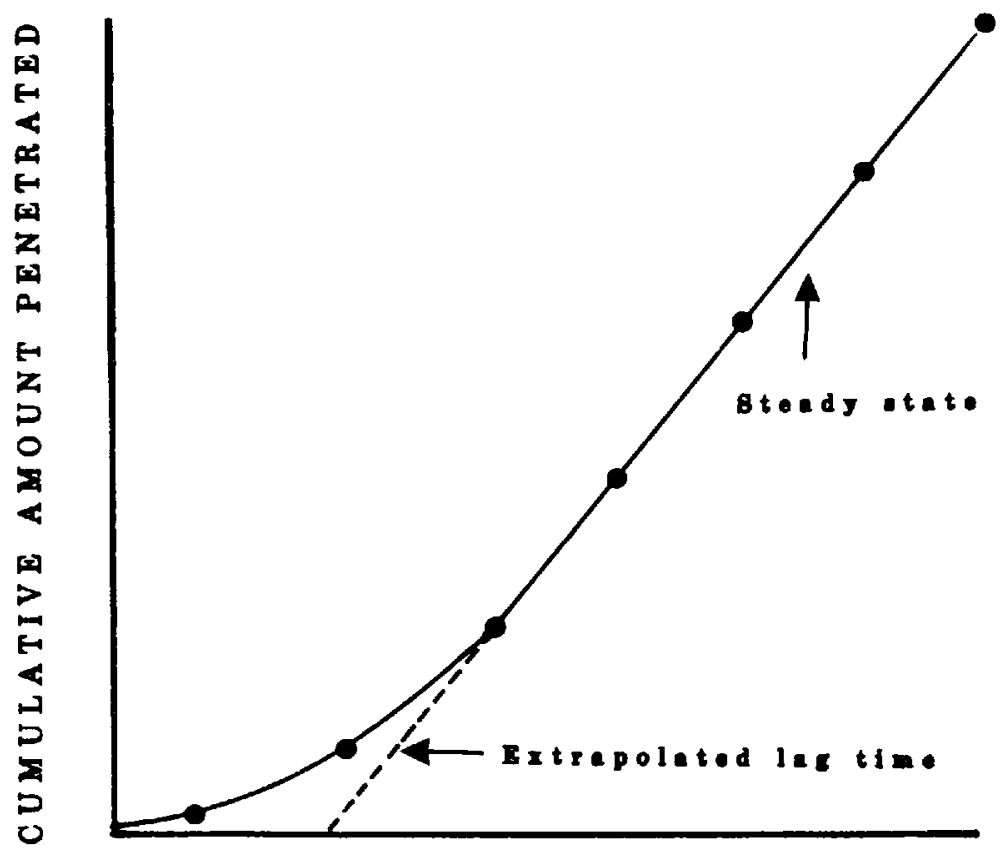

TI M E

Fig. 2. Schematic representation of the standard permeation profile (amount penetrated through the membrane as a function of time), which is obtained using a two-compartment diffusion cell. The intercept of the steady-state line (equation 2 in the text) is the lag time (equation 4 in the text).

$$
\mathbf{M}_{\mathrm{t}}=\frac{\mathrm{K} \cdot \mathrm{D}_{\mathrm{sc}} \cdot \mathrm{C}_{\mathrm{s}}}{\mathrm{h}_{\mathrm{sc}}}\left(\mathrm{t}-\frac{\mathrm{h}_{\mathrm{sc}}{ }^{2}}{6 \mathrm{D}_{\mathrm{sc}}}\right)
$$

In effect, equation 2 describes a situation in which the amount penetrating per unit time is constant, the steady-state condition. The steady-state flux, $\mathrm{dM}_{\mathrm{t}} / \mathrm{dt}$, is readily obtained from equation 2 by differentiating $\mathbf{M}_{\mathrm{t}}$ with respect to $\mathrm{t}$, i.e.:

$$
\frac{\mathrm{dM}_{\mathrm{t}}}{\mathrm{dt}}=\frac{\mathrm{K} \cdot \mathrm{D}_{\mathrm{sc}} \cdot \mathrm{C}_{\mathrm{s}}}{\mathrm{h}_{\mathrm{sc}}}
$$

When the steady-state line is extrapolated to the time axis, the value of $t$ at $M=0$ is found to be:

$$
t_{L}=\frac{h_{s c}^{2}}{6 D_{s c}}
$$

This intercept, $t_{\mathrm{L}}$, is referred to as the lag time. In essence, it is a measure of the time it takes for the permeant's concentration gradient to become stabilized across the membrane. In 
principle (with the simplest of membranes), the diffusivity can be calculated from $t_{L}$ providing the membrane thickness is known. With a complex membrane such as the skin, only an effective value of the diffusivity is obtained.

The essential meanings of equations 1 through 4 can be considered. Equation 4 tells us that the lag time for penetration becomes longer as the effective diffusivity, $\mathrm{D}_{\mathrm{se}}$, becomes smaller (irrespective of how it is calculated). Normally one wants the lag time to be a negligible fraction of the projected time of use of a transdermal system. If the prescribed period of application is to be a day, as it is with current nitroglycerine patches, the lag time should be no more than a tenth of a day or 2-3 hours at the most. Any lag time longer than this raises questions about the utility of the patch, for the patch will not be delivering drug over a significant part of the time it is in use. Consequently, the attainment and maintenance of even blood levels will be next to impossible. In vitro diffusion cell studies suggest that the values of $D_{\mathrm{sc}}$ for the stratum corneum (one uses the stratum corneum's 10- $\mu \mathrm{m}$ thickness in the calculation) range from $10^{-9}$ down to $10^{-12} \mathrm{~cm}^{2} / \mathrm{sec}$ [Scheuplein, 1978; Scheuplein and Bronaugh, 1983]. At the larger diffusivities, lag times of several minutes are expected. On the other hand, multiple-day lag times are suggested by the smallest reported diffusivities. In our actual experience, diffusional lag times range from several hours to over half a day for nonelectrolyte drugs having molecular weights of 200 daltons or more. Moreover, the more polyfunctional and polar a compound is, the longer its lag time seems to be. For example, lag times across cadaver skin membranes for the permeation of fentanyl and sufentanil, both narcotics of low polarity based on partitioning data, have consistently been less than 3 hours. In contrast, lag times for morphine and hydromorphone, both relatively polar narcotics, have consistently exceeded 6 hours, well over than twice those of fentanyl and sufentanil. This alone poses a problem with respect to their transdermal delivery. Clearly, the first obstacle to transdermal delivery is overly long lag times. Fortunately, in vitro diffusion cell methods appear to be able to indicate when this is going to be a problem.

Equation 1 indicates that, providing enough time is allowed to elapse, a steady state in permeation is obtained. The rate of delivery in the steady state is described by equation 3 . The steady state arises in part because delivery is from a reservoir of constant thermodynamic activity. The development of a steady state also requires the barrier properties of the skin membrane to be invariant over the lifetime of patch use (or over the duration of a diffusion cell experiment). For the purpose of analysis, we will assume the skin membrane is stable, in which instance equation 3 tells us that the per unit area rate of delivery is proportional to the product of the saturation concentration, $C_{s}$, diffusivity, $D_{s c}$, and the partition coefficient, $K$, as well as inversely proportional to the thickness of the membrane, $h_{s c}$. The product, $K \cdot C_{s}$, yields the saturation concentration in the surfacemost structure of the stratum corneum ${ }^{1}$ and is, at least theoretically, independent of the nature of the medium used in the reservoir. The process is driven by the activity of the solid state; the gradient across the skin directly reflects this activity. For a complex membrane such as the skin, derived values for $D_{s c}, K$, and $h_{s c}$ have magnitudes that depend on assumptions about the nature of the membrane. Since they cannot be experimentally disentangled, they are commonly lumped together in a single term. One thus arrives at:

$$
\frac{\mathrm{dM}_{\mathrm{t}}}{\mathrm{dt}}=\mathrm{P} \cdot \mathrm{C}_{\mathrm{s}}=\text { constant }
$$

\footnotetext{
${ }^{1}$ A mechanism of permeation of a drug involving diffusion of the drug across the stratum corneum, the so-called transepidermal pathway, is assumed. However, the principles set forth are general with the assumption having no practical consequence on the analysis.
} 
where $\mathbf{P}$ is the mass transfer coefficient or "permeability coefficient." The total flux in the steady state is therefore:

$$
\left(\frac{\mathrm{dM}_{\mathrm{t}}}{\mathrm{dt}}\right)_{\text {Total }}=\mathrm{V}\left(\frac{\mathrm{dC}_{\mathrm{t}}}{\mathrm{dt}}\right)_{\text {Total }}=\mathrm{A} \cdot \mathrm{P} \cdot \mathrm{C}_{\mathrm{s}}
$$

where now the area, A, is explicitly considered. It is obvious that, in any designated small increment of time, the total amount of drug that permeates the membrane, $\mathbf{d M}_{\mathbf{t} \text {,Total }}$, is equal to the volume of the collecting phase, $\mathrm{V}$, times the change in concentration of this phase, $\mathrm{dC}_{\mathrm{t}}$. It is these forms of equation 3 that are used to calculate permeability coefficients from data gathered in in vitro studies. This is possible because the area available for diffusion in the diffusion cell and the solubility or, more generally, concentration of the drug in the medium applied to the skin mounted in the diffusion cell are independently measured and known prior to starting an experiment. The in vitro experiment is begun, and the flux in the steady state is measured. From these, one calculates the proportionality constant between flux and the product of area and concentration. This is what the permeability coefficient actually is. It takes a value that is uniquely dependent on: 1) the chemical structure of the drug; 2) the physicochemical nature of the medium of application; and, of course, 3) the physicochemical nature of the membrane.

When the membrane and medium of application are fixed, differences in permeability coefficients through a family of drugs are strictly related to the differences in chemical structures of the drugs. Clearly, the larger the value of $\mathrm{P}$, the greater the flux of the compound, all other factors being equal. According to Flynn [1985], permeability coefficients for hairless mouse skin determined from saline (or isotonic aqueous buffer) range from about $10^{-5}$ to about $10^{-2} \mathrm{~cm} /$ hour, with indications that they take levels ten times smaller in human epidermis on the lower side of the range. Using $1 \mathrm{mg} / \mathrm{ml}$ as a reference concentration ${ }^{2}$ and the maximum permeability coefficient of $10^{-2} \mathrm{~cm} /$ hour, one calculates that $20 \mu \mathrm{g}$ of drug passes through the skin membrane per hour per $\mathrm{cm}^{2}$. This amounts to about $0.5 \mathrm{mg} / \mathrm{cm}^{2} /$ day. By chance, this is almost exactly the amount of nitroglycerine that can be induced to diffuse through the skin. Nitroglycerine is a liquid at room temperature. It also has the requisite physicochemical properties to place it at the high end of the permeability scale. Since it has these ideal properties for transdermal delivery, it would appear that a flux of one half mg per day per $\mathrm{cm}^{2}$ should be near the upper limit of attainable flux. At the lower end of the permeability coefficient scale, the computed daily delivery is, for all practical purposes, negligible unless permeation is from very high concentrations of a drug. ${ }^{3}$ Thus, a small permeability coefficient sets the second physical limitation on transdermal delivery.

Equation 5 indicates that a second general factor determining flux is the concentration of the drug in the medium of application. As pointed out, the limiting mass transfer rate is seen when permeation is from a saturated solution. In general, supersaturation leads to a metastable situation and cannot be relied upon. In order to use equation 5, one must know the solubility of the drug in the medium that was used in the assessment of the permeability coefficient. Almost invariably the solubility of interest will be the solubility in water, as the vast majority of published permeability coefficients for the skin have been determined using aqueous media.

\footnotetext{
${ }^{2}$ Note that only relatively hydrophobic drugs have permeability coefficients as large as $1 \times 10^{-2}$ $\mathrm{cm} /$ hour, and these rarely have solubilities in water as high as $1 \mathrm{mg} / \mathrm{ml}$.

${ }^{3}$ However, large, polyfunctional, polar compounds tend to be highly crystalline and, consequently, none too soluble in any phase.
} 
Consequently, any scheme for estimating permeability coefficients will of necessity be referenced to data generated in watery media.

Several completely separate factors have a bearing on solubility, including the ease of dissociation of a molecule from its crystal and the degree of interaction of dissolved solute molecules with the solvent. High-melting, hard crystalline materials with large enthalpies of fusion are predictably less soluble than soft, low-melting crystals, everything else being equal. It turns out that hydrophobic molecules have low intracrystalline cohesiveness and, according to this factor, tend to be relatively soluble. However, the net negative free energy of solution of hydrophobic molecules in water is small, meaning such molecules have low aqueous solubilities. On the other hand, hydrophobic crystalline organics tend to be highly soluble in nonpolar media, including the conduit phases of the skin. As a consequence, they have large permeability coefficients, which tend to more than compensate for their low water solubilities ${ }^{4}$ For this reason, high fluxes are seen with moderately hydrophobic drugs.

Molecules containing multiple hydrogen bonding centers and/or strong dipoles are generally high-melting because of strong intracrystalline self-association. While such molecules react strongly with water, imparting a degree of solubility in this solvent, they do not dissolve substantially in organic phases, because of the exaggerated cohesive energy difference between polar solute and nonpolar solvent. Consequently, partitioning into the lipoidal conduit phases of the skin is minimal, and mass transfer rates are low even from their saturated solutions. It is clear then that low solubility is the third physical obstacle to transdermal delivery.

\section{Transdermal Delivery Rate Projection}

The above discussion indicates that the transdermal delivery rate of a drug can be forecast providing one knows the drug's solubility in and permeability coefficient from a given solvent medium. It further suggests that the logical solvent medium on which to base such projections is water. One can often find the aqueous solubilities of drugs in compendia or the research literature. When the solubility of a compound of interest is not available, it is a reasonably straightforward task to determine it, generally a 1 -day experiment, providing there is an ample supply of drug. In doing so, one has to account for the ionization of weak electrolytes, so that the correct solubility of the drug can be assigned to the solid state representing the nonelectrolyte form, for it will be the partition coefficient of the free form that will be used to estimate a permeability coefficient. In principle, one also should be able to estimate solubilities by one of several empirical methods. However, it has been our experience that solubilities determined by the best of such methods, for instance, those based on the melting points of drugs and their octanol/water partition coefficients [Yalkowsky, 1981], are not sufficiently reliable for predictive purposes.

Permeability coefficients are neither prevalent in the literature nor so easily determined; there is no immediately obvious means of estimating them that does not involve permeation experiments. However, on careful examination of the literature, one finds prediction-useful relationships between the permeability coefficients of drugs and their partitioning tendencies. Flynn [1985] has discussed how the permeability coefficients of test compounds through hairless mouse skin relate to the ether/water partition coefficients of the compounds. The reported data are displayed in Figure 3. It can be seen that compounds having molecular weights greater than 200 daltons all seem to lie on a sigmoidal line stretching over ten logarithmic orders of partition coefficient. The upper limit on the values is slightly above $10^{-2}$

\footnotetext{
${ }^{4}$ One can view this another way. Although the solubility of a hydrophobic compound in water is low, the product of this solubility and the partition coefficient into the skin is likely to be large, meaning that the drug's solubility in the surface structure of the skin is large. This goes hand in hand with the presumed permeability coefficient.
} 


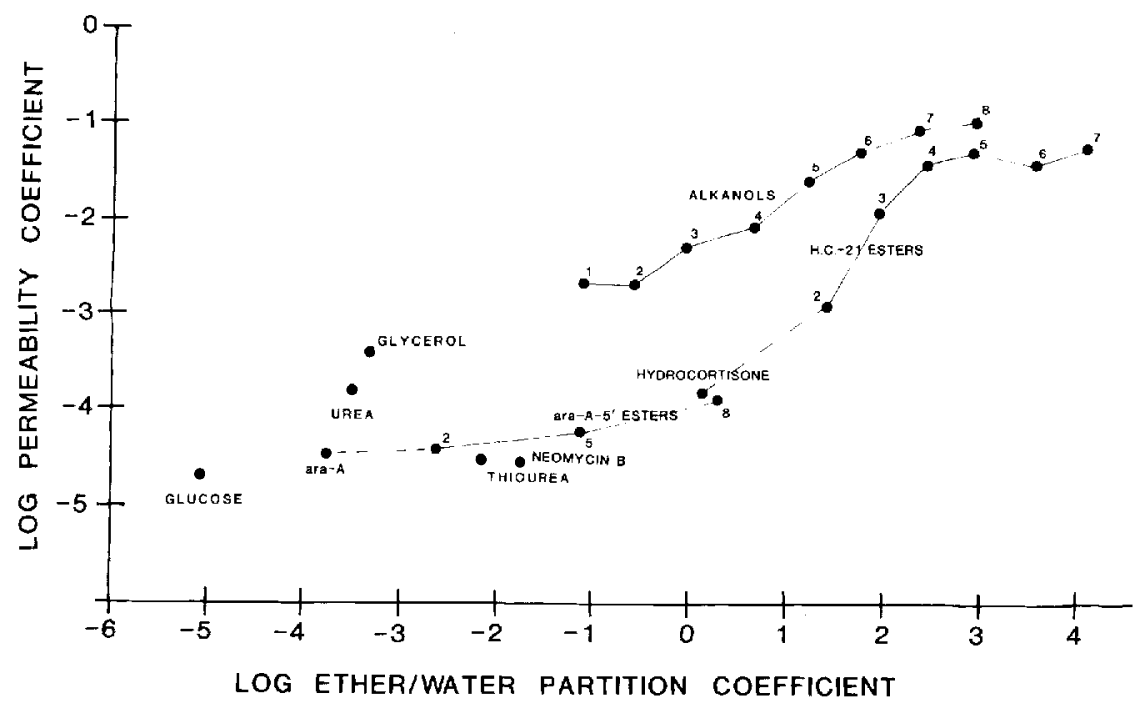

Fig. 3. Plot showing how the permeability coefficients of a number of test compounds and drugs through hairless mouse skin relate to their ether/water partition coefficients. For compounds of molecular weight $>200$ daltons, all data fall on or reasonably close to a sigmoidal line spanning about ten log orders of magnitude of partition coefficients (X-axis) and over three log orders of permeability coefficients (Y-axis).

$\mathrm{cm} /$ hour and is attained at ether/water partition coefficients greater than $500\left(\log _{10}=2.7\right)$. Over the range of partition coefficients from $0.01\left(\log _{10}=-2\right)$ to $100\left(\log _{10}=+2\right)$, the permeability coefficients increase by three logarithmic orders. Finally, compounds with $\log$ (permeability coefficients) less than -2 all fall on a line just above $P=10^{-5} \mathrm{~cm} / \mathrm{hour}$. We have found that these permeability coefficients fit to a similar general pattern on an octanol/water partition coefficient scale.

\section{Permeability Analysis-Top 200 Drugs}

To put the principles outlined above to a stringent test, it was decided to apply them to an evaluation of the transdermal delivery potentials of the drugs in the top 200 new prescription drug products of $1985^{5}$. The top 200 list is actually one of products, not chemical entities, and is full of entity redundancy. Moreover, it was found that the necessary information to make projections was available for only $22(\approx 15 \%)$ of the actual chemical entities on the list. Twenty-two compounds are nevertheless a sufficient cache of drugs to give fair assessment of the utility of any estimative approach.

Establishing the daily transdermal drug requirement was the first step taken toward estimating the transdermal potentials of the indicated drugs. It was reasoned that this would be equal to the total 24-hour oral dose adjusted downward for oral first-pass loss. Oral dosages were drawn from standard compendia [Huff, 1986; Boyd, 1981]. First-pass information was mainly found in Goodman and Gilman's classic pharmacology text [Goodman et al., 1985]. No data on oral first-pass extraction could be found for 8 of the 22 compounds listed in Table 1. No attempt was made to account for conceivable but relatively improbable metabolism in the skin (see the final paragraphs of this essay). The formula for computing the dose was:

\footnotetext{
${ }^{5}$ A manuscript by B. H. Stewart, G. L. Flynn, N. Sheth, and S. D. Roy entitled Physiochemical assessment of the transdermal delivery potentials of drugs is in preparation.
} 
TABLE 1. Selected Drugs From the Top 200 (1985 List) Used to Test the Method of Assessing Transdermal Delivery Potentials

\begin{tabular}{|c|c|c|c|c|c|}
\hline Chemical name & $\begin{array}{c}\text { Permeability } \\
\text { coefficient }^{\mathrm{a}} \\
\left(\mathrm{cm} / \mathrm{hr} \times 10^{3}\right)\end{array}$ & $\begin{array}{c}\text { Aqueous } \\
\text { solubility }^{\mathrm{b}} \\
\text { (mg/ml) }\end{array}$ & $\begin{array}{c}\text { Total daily } \\
\text { transepidermal } \\
\text { dose }^{\mathrm{c}, \mathrm{d}} \\
(\mathrm{mg})\end{array}$ & $\begin{array}{c}\text { Estimated } 24-\mathrm{hr} \\
\text { transepidermal } \\
\text { delivery } \\
\left(\mathrm{mg} / \mathrm{cm}^{2}\right)\end{array}$ & $\begin{array}{c}\text { Patch } \\
\text { size } \\
\left(\mathrm{cm}^{2}\right) \\
\end{array}$ \\
\hline Acetylsalicylic acid & 3.25 & 3.3 & 1,000 & 0.0257 & - \\
\hline Allopurinol & 0.016 & 0.48 & 250 & 0.00018 & - \\
\hline Amoxicillin & 1.48 & 4.0 & $\overline{700}$ & 0.142 & - \\
\hline Chlorpropamide & 0.036 & 2.2 & 225 & 0.0019 & - \\
\hline Clonidine & 3.82 & 30.0 & 0.2 & 2.75 & $<1$ \\
\hline Codeine phosphate & 0.41 & 8.3 & 120 & 0.082 & - \\
\hline Dexamethasone & 19.6 & 0.1 & 4 & 0.047 & 85 \\
\hline Erythromycin & 20.0 & 2.0 & 350 & 0.960 & - \\
\hline Erythromycin estolate & 20.0 & 0.024 & 350 & 0.0115 & - \\
\hline Estrone sulfate & 20.0 & 0.03 & 1.25 & 0.0144 & 87 \\
\hline Haloperidol & 20.0 & 0.014 & $\overline{2.0}$ & 0,0067 & - \\
\hline Hydorcortisone acetate & 5.27 & 0.01 & 200 & 0.0013 & - \\
\hline Lorazepam & 20.0 & 0.08 & $\overline{4}$ & 0.0384 & 104 \\
\hline Methyldopa & 0.2 & 10 & 150 & 0.048 & $\ldots$ \\
\hline Nicotine & 2.96 & 100.0 & 20 & 7.1 & 2.8 \\
\hline Nitroglycerine & 20.0 & 1.25 & 2.5 & 0.6 & 4.2 \\
\hline Penicillin V & 20.0 & 0.25 & 750 & 0.12 & - \\
\hline Piroxicam & 6.21 & 0.01 & 20 & 0.0015 & - \\
\hline Phenytoin & 20.0 & 0.013 & $3 \overline{00}$ & 0.0062 & - \\
\hline Tetracycline & 0.014 & 1.70 & 1,000 & 0.00057 & - \\
\hline Theophylline & 0.19 & 8.3 & 385 & 0.038 & - \\
\hline Trimethoprim & 1.63 & 0.4 & 2,000 & 0.0156 & - \\
\hline
\end{tabular}

aPermeability coefficients were estimated from the octanol/water partition coefficient using the formula found in the text.

${ }^{b}$ All values for the solubilities were obtained from the literature and were rounded to a comfortable magnitude.

'The daily transdermal dose is equal to the daily oral dose $\times(100-\%$ extracted via first pass $)$.

No data on first-pass extraction could be found for the compounds with underlined values.

"See equation 8 in the text.

$$
\mathrm{D}_{24} \mathrm{hr} \text {, transdernal }=\mathrm{D}_{24 \mathrm{hr}, \text { oral }} \times(100-\% \text { extracted first-pass })
$$

Each computed daily transdermal dose, $\mathrm{D}_{24} \mathrm{hr}$, transdermal, was rounded to the nearest whole number; the doses are given in column 4 of Table 1 .

The literature was then scoured for the aqueous solubilities of the listed compounds. The Merck Index [Windholz, 1983] was relied on heavily for these. The paucity of solubility information on the compounds in the top 200 list limited the number of projections that could be made. Very few reliable, experimental, aqueous solubilities were found. Solubilities obtained from the literature are provided, in units of $\mathrm{mg} / \mathrm{ml}$, in columns of Table 1 .

With the exceptions of nitroglycerine and clonidine, little if any skin permeation data appear in the literature for the 22 test compounds. Thus all but two permeability coefficients had to be estimated. Moreover, given the purpose of the exercise, the permeability coefficients of nitroglycerine and clonidine were estimated exactly like the others so they might serve to check the method. Permeability coefficients were also estimated for estradiol, fentanyl, and scopolamine so that projected delivery rates could be compared with experimental data (Table 2). Therefore a means had to be devised to assign permeability coefficients to the drugs. It has 
TABLE 2. Transdermal Delivery Calculations on Estradiol, Fentanyl, and Scopolamine

\begin{tabular}{lccccc}
\hline $\begin{array}{l}\text { Chemical } \\
\text { name }\end{array}$ & $\begin{array}{c}\mathrm{C}_{\mathrm{s}} \\
(\mathrm{mg} / \mathrm{ml})\end{array}$ & $\begin{array}{c}\mathrm{P} \text {, actual } \\
(\mathrm{cm} / \mathrm{hr}) \times 10^{3}\end{array}$ & $\begin{array}{c}\mathrm{P}, \text { estimated } \\
(\mathrm{cm} / \mathrm{hr}) \times 10^{3}\end{array}$ & $\begin{array}{c}\mathbf{M}_{24, \text { actual }} \\
\left(\mathrm{mg} / \mathrm{cm}^{2} / \text { day }\right)\end{array}$ & $\begin{array}{l}\mathbf{M}_{24, \text { estimated }}{ }^{\mathrm{b}} \\
\left(\mathrm{mg} / \mathrm{cm}^{2} / \mathrm{day}\right)\end{array}$ \\
\hline Estradiol $^{\mathrm{c}}$ & 0.003 & 5.2 & 20.0 & 0.0004 & $0.0014(3.6)$ \\
Fentanyl $^{\mathrm{c}}$ & 0.20 & 10.0 & 20.0 & 0.0048 & $0.0096(2)$ \\
Scopolamine $^{\mathrm{c}}$ & 75.0 & 0.05 & $3.5^{\mathrm{d}}$ & 0.09 & $6.3(70)$ \\
\hline
\end{tabular}

${ }^{a}$ Estimated by the algorithm based on $\mathrm{K}_{\mathrm{octano} / \text { water }}$ partition coefficients outlined in the text of the article. The partition coefficients were found in Hansch and Leo [1979].

bIn parentheses: projected 24-hr delivery/experimentally determined 24-hr delivery.

${ }^{c}$ Experimental data are from Michaels et al. [1975]. Yet to be published data on the permeability coefficient and solubility of fentanyl from these laboratories are in very close agreement with the Michaels et al, values.

${ }^{\mathrm{d}}$ The reported $\log _{10}$ (partition coefficient) of 1.26 on which this permeability coefficient value is based is footnoted as "approximate." The value appears inconsistent with the high aqueous solubility of the compound and its reported solubilities in apolar organic solvents such as benzene and pet ether. The mineral $\log _{10}$ (oil/water) partition coefficient is -1.59 [Michaels et al., 1975]; the $\log _{10}$ (heptane/water) partition coefficient is -2.26 [Hansch and Leo, 1979].

been previously mentioned and is well established that permeability coefficients correlate well with in vitro partition coefficients [Scheuplein, 1965; 1967; 1983; Michaels et al., 1975; Flynn, 1985]. Because of the existing extensive compilations of octanol/water partition coefficients [Hansch and Leo, 1979], the decision was made to scale the permeability coefficients of the compounds to their $\mathrm{K}_{\text {octano//water }}$ partition coefficients. Based on a roughed-out plot of the permeability coefficients of certain compounds against their octanol/ water partition coefficients, the following algorithm was developed to estimate permeability coefficients. For compounds with $\log _{10}\left(\mathbf{K}_{\text {octanol/water }}\right)<-2.301$, a lower limit of $1 \times 10^{-6}$ $\mathrm{cm} /$ hour was assigned to the permeability coefficient. In this region the compounds were considered polar enough to preferentially pass through the aqueous regime of the stratum corneum [Flynn, 1985]. For compounds with $\log _{10}\left(\mathrm{~K}_{\text {octanol/water }}\right)$ values lying between -2.301 and +2.000 , the permeability coefficients were determined from the empirically derived relationship:

$$
\log _{10}(\mathrm{P})=\log _{10}\left(\mathrm{~K}_{\mathrm{octanol} / \mathrm{water}}\right)-3.698
$$

It is important that the steepness of this line and the nature of its placement on the octanol/water axis, which, as stated above, involved fitting the line through several experimental data points, leaves considerable doubt about the accuracy, and therefore the usefulness, of permeability coefficients estimated over this span of partition coefficients. For compounds with $\log _{10}$ $\left(K_{\text {octanol/water }}\right)$ values $>2.000$, an upper limit permeability coefficient of $1 \times 10^{-2} \mathrm{~cm} /$ hour was assigned. Compounds fitting into this partitioning range werc considered sufficiently nonpolar for their permeation rates to be controlled within aqueous strata of the epidermis and dermis [Flynn, 1985].

In some instances, multiple values for the octanol/water partition coefficients of compounds on the top 200 list have been reported; when there was a lack of agreement between the values, the value that intuitively appeared to be the most reasonable was arbitrarily chosen. Permeability coefficients were then computed (units of $\mathrm{cm} / \mathrm{hour}$ ) and rounded to a comfortable number; they are given in column 2 of Table 1 .

Using the solubilities and permeability coefficients, the cumulative amount of drug that can be made to pass through a $1-\mathrm{cm}^{2}$ area of skin from a saturated solution in a day, $\mathbf{M}_{24 \mathrm{hr}}$, in milligrams was calculated from: 
$\mathrm{M}_{24 \mathrm{hr}}=\mathrm{P}_{\text {estimated }} \cdot \mathrm{C}_{\mathrm{s}} \cdot 24$

This equation will be recognized as an integrated form of equation 5 . Column 5 of Table 1 gives the estimated 24-hour amounts. Only when within $\approx 100$-fold of the total daily transdermal requirement (ratio of daily dose to $M_{24 \mathrm{hr}} \approx 100$ ) were patch sizes that would be adequate to deliver the daily dose computed (last column in Table 1).

It can be seen from Table 1 that the method of estimation suggests that it is physically possible to deliver clonidine and nitroglycerine by the transdermal method and, moreover, that clonidine can be offered as a tiny transdermal device. Considering that transdermal products are available for both drugs and that clonidine's patch is indeed small $\left(\approx 2.5 \mathrm{~cm}^{2}\right)$, all three of these projections have been borne out. Nicotine is also projected to be a good transdermal candidate. Developments in several laboratories have demonstrated this to be true. However, and more quantitatively, the projected daily deliveries of all three of these drugs appear overestimated by three- to five-fold.

The analysis further suggests that dexamethasone, estrone sulfate, lorazepam, and possibly haloperidol (ratio $\approx 300$ ) may be borderline candidates for the transdermal method. Dexamethasone is not a transdermal candidate for systemic purposes, as it is a corticosteroid well suited to oral administration. However, the drug is topically active. At this writing, there are strong indications that estrone sulfate is being readied for transdermal use. Lorazepam also seems to have genuine transdermal possibilities, but the same can be said for other highly potent benzodiazepines. Whether haloperidol is a true candidate has to be researched, but the possibility cannot be discounted. No other drug in the list is even close to meeting the minimal specifications for transdermal delivery. Some fail because of high daily doses, while others fall short because of intrinsically low skin permeabilities.

The predictive approach can be further challenged by comparing other drugs with the top 200 list. Predicting the fluxes of drugs currently available or being developed for transdermal administration provides an interesting test. Therefore, $\mathbf{K}_{\text {octanul/water }}$-determined permeability coefficients were estimated for estradiol, fentanyl, and scopolamine [Michaels et al., 1975] ${ }^{6}$ These are found in Table 2 alongside the known values. The estimated permeability coefficients for both estradiol and fentanyl are within factors of four of their reported values, certainly an acceptable level of agreement, and consequently the $\mathrm{M}_{24}$ values, actual and projected, are equally close because the same solubility was used for the $M_{24}$, actuai and $\mathbf{M}_{24}$, estimated calculations. The predictive method seems to break down with scopolamine, however. The estimated permeability coefficient is 70 times the experimental one; consequently, the estimated daily flux is also 70 times too large. While the octanol/water partition coefficient for scopolamine is suspect (see footnotes to Table 2), we suspect the real problem with the prediction for scopolamine results from a discrepancy in the relationship between octanol/water partitioning and skin partitioning. In other words, the fundamental platform upon which the predictive method is based seems itself to be weakening. The permeability is overstated by over an order of magnitude. Since the error of estimation overstates rather than understates the potential of scopolamine, the compound would not be rejected out of hand as a transdermal candidate, however. We suspect further breakdowns as this might occur with highly water-soluble compounds that are also highly soluble in oils of intermediate polarity, for it is for just such compounds that the correlation between octanol/water partitioning and skin/water partitioning is likely to languish.

We are currently compiling a broader data base to tighten the estimative method and reveal where the method might fall short of expectation. The tendency to overestimate delivery at the upper range of partition coefficient suggests that the upper limit value should be cut,

${ }^{6} \mathrm{Also}$ including date for fentanyl from unpublished research done in these labs. 
possibly by a much as four-fold (to $5 \times 10^{-3} \mathrm{~cm} / \mathrm{hour}$ ). As more data are gathered, the best placement for the line at intermediate polarity will become more evident. It almost doesn't matter where the lower limit line is set, as only in the rarest of circumstances will a drug in the high polarity regime make the grade transdermally.

Frankly, we are encouraged by two aspects of the above analysis, first, that the transdermal delivery potentials of so many compounds can be identified simply from physical parameters that are typically gathered during preformulation exercises, and second, that no good candidates "fell through the cracks." Presently, the limiting factors in making predictions are the lack of availability of aqueous solubilities of the drugs and the disparities in reported partition coefficients. Although we have placed the assessment of transdermal delivery potential in a tighter quantitative framework than ever before, in reality we offer nothing totally new concerning the factors that make transdermal delivery a possibility [Shaw and Chandrasekaran, 1978; Chien, 1982; Cleary, 1983; Karim, 1983; Wester, 1985; Guy and Hadgraft, 1985; Good, 1986; Walters, 1986]. It takes potent drugs! Consider that the identified transdermal candidate with the highest dose of those considered, nicotine, only requires an estimated $20 \mathrm{mg}$ to be delivered over the course of a day; this is high, as many, if not most, candidates for the method of delivery, proven or suspected, have daily doses that are measured in whole micrograms, not milligrams. Note also that all the promising candidates had permeability coefficients at or near the established limiting value, meaning that all are lipophilic. The idea that lipophilic drugs are the most likely drugs to "make it"' isn't fresh. But why this dependency on lipophilicity? Here we point out that the most productive routes through (or around) the principal barrier layer of skin, the stratum corneum, are lipoidal, which favors solution of such species. This fact alone ties the opening discussion to the predictive method. Moreover, lipoidal compounds exhibit low molecular cohesiveness, including intracrystalline cohesiveness. Therefore they melt at lower temperatures and consume less total energy in melting per mole than do polar organics. This makes them generally more soluble, although obviously not so in water. This adds to the suitability of lipophilic drugs for transdermal delivery, as they are not only more relatively soluble but also more absolutely soluble in the skin's critical diffusion conducting phases. The absolute solubility issue and its relationship to low molecular cohesiveness ties in other parameters, like the melting points of drugs, that have been used in a priori estimations of transdermal delivery potentials.

\section{BIOLOGICAL CONSIDERATIONS IN TRANSDERMAL DELIVERY Irritancy and Allergy Issues}

Even when a drug has the requisite potency and permeability for transdermal delivery, one is not sure the drug will make it to the marketplace in transdermal form because toxic reactions within the skin resulting from application of the transdermal delivery system can still scuttle the development project. Local irritancy associated with the drug and/or its delivery system is a prime concern. The potential of any drug to induce an allergic response in the skin is also worrisome. Both factors have caused a good deal of grief and disappointment along the transdermal development path, often at the 11 th hour when the highest price is paid for setback and failure. Part of the problem is that human irritant responses, allergic sensitivities, and photosensitivities are not actually predictable. Standard toxicity tests in animals are done in the course of development of a transdermal device of course, but, unfortunately, such tests have a poor record of extrapolation to the human condition. When no problems are encountered in the animal models, the project team may proceed with a drug's development falsely confident and without further questioning. That the drug in question may already be in use by the oral route without a significant incidence of skin toxicity only adds to the false sense of security. It then comes as a shock when the exhaustively physically tested and fully developed drug delivery system is not well tolerated in the clinic. Clearly, irritancy and allergy set a most stringent constraint on transdermal delivery. Because of the stakes involved, it behooves the 
development team to obtain an early, direct reading of the human skin toxicity of a drug. This requires strategic arranging of the sequencing of development events to bring human skin tolerance testing into the foreground.

It should be pointed out that there is a straightforward reason why drugs that have clean dermatotoxicity records when used orally and parenterally can run into toxicity problems when topically deployed. Transdermal delivery leads to drug accumulations in the skin directly beneath a patch that are many times higher than are ever achieved in the skin through systemic administration of that drug. Irritancy (particularly) and allergic responses are known to be concentration-dependent phenomena; topical administration has the potential of raising the tissue concentrations of a drug (or other component of the transdermal system) above the threshold for response. While it appears possible to modulate irritant responses, for instance by designing a larger patch that delivers less drug per unit area, it seems that once the skin of a patient is sensitized to a drug, that particular drug is forever contraindicated by the topical route for that patient.

\section{Metabolism During Transit Through the Skin}

Another biological factor that can affect transdermal delivery is metabolism of the drug in the skin coincident with its diffusive passage across the skin. It is conceivable that little intact drug might reach the systemic circulation. Thus local first-pass metabolism at least has the potential to act as another constraint on transdermal delivery. However, all current experience suggests that this is not a general problem. For one thing, the skin has far less capacity to metabolize drugs than have either the liver or the gastrointestinal mucosa, the latter of which routinely synthesizes glucuronides and other adducts as drugs are absorbed. Moreover, it appears from early observations in these labs that human skin has less capacity to enzymatically alter drugs than do the skins of the rat and mouse. In particular, human skin seems to have a low esterase activity. Other enzyme activities of the skin, human or otherwise, seem to be substrate-specific and purposeful, and unlikely to cause problems with an exogenous compound.

Enzymatic inactivation of a transdermally administered drug in the skin need not be dismissed out of hand because of its improbability, for one can easily experimentally establish whether or not concern should exist over the issue. The presence or absence of metabolites can be demonstrated in in vitro diffusion cell experiments. Such studies also suggest the extent of metabolism that will occur as the drug is absorbed. Also, the drug can be incubated with skin homogenates to check for metabolism in the presence of all relevant enzyme cofactors. If metabolism is possible at all, it will normally show up in such experiments.

\section{REFERENCES}

Boyd, J. R. (ed): “Drug Facts and Comparisons,” 1982 edition. St. Louis: Facts and Comparisons, 1981.

Chien, Y. W.: Transdermal controlled-release drug administration. In: "Novel Drug Delivery Systems-Fundamentals, Development Concepts, Biomedical Assessments. New York: Marcel Dekker, Inc., 1982, pp. 149-217.

Cleary, G. W.: Transdermal controlled release systems, In Langer, R. S. and Wise, D. L. (eds): "Medical Applications of Controlled Release"' (Vol. I). Boca Raton, FL: CRC Press, 1983, pp. $203-251$.

Elias, P., Grayson, S., Lampe, M. A., Williams, M. L., and Brown, B. E.: The intercorneocyte space. In: Marks, R. and Plewig, G. (eds): “Stratum Corneum." New York: Springer-Verlag, 1983, pp. 53-67.

Flynn, G. L.: Mechanism of percutaneous absorption from physicochemical evidence. In Bronaugh, R. L. and Maibach, H. I. (eds): "Percutaneous Absorption." New York: Marcel Dekker, Inc., 1985, pp. $17-42$.

Goldsmith, L. A.: The epidermal cell periphery. In Goldsmith, L. A., (ed): "Biochemistry and Physiology of the Skin" (Vol I) New York: Oxford University Press, 1983, pp. 184-199. 
Good, W. R.: Transdermal delivery systems, Med. Device Diagn. Ind. Feb. 1986: 35-42, 1986.

Goodman, A. G., Gilman, L. S., Rall, T. W., and Murad, F.: "Goodman and Gilman's The Pharmaceutical Basis of Therapeutics"' 7th edition. New York: Macmillan Publishing Co., 1985, pp. $1668-1733$.

Guy, R. H. and Hadgraft, J.: Transdermal drug delivery: The ground rules are emerging, Pharm. Int., May 1985:112-116, 1985.

Hansch, C. and Leo, A.: "Substituent Constants for Correlation Analysis in Chemistry and Biology." New York: John Wiley and Sons, 1979.

Huff, B. B. (managing ed): "Physician's Desk Reference." Oradell, NJ: Medical Economics Co., Inc., 1986.

Karim, A.: Transdermal delivery systems. In "Drug Delivery Systems, Proceedings of an International Conference" (Special Issue of Pharmaceutical Technology). Springfield, R: Aster Publishing Co., 1983 , pp. 28-32.

Katz, M. and Poulsen, B. J.: Absorption of drugs through the skin. In Brodie, B. B. and Gillette, J. (eds): "Handbook of Experimental Pharmacology, New Series"' (Vol. 28). New York: Springer-Verlag, 1971, pp. 103-174.

Kligman, A. M.: Perspectives and problems in cutaneous gerontology, J. Invest. Dermatol. 73: 39-46, 1979.

Michaels, A. S., Chandrasekaran, S. K., and Shaw, J. E.: Drug permeation through human skin: Theory and in vitro experimental method, A.1.Ch.E.J. 21: 985-996, 1975.

Odland, G. F.: Structure of the skin. In Goldsmith, L. A. (ed): "Biochemistry and Physiology of the Skin" (Vol. I). New York: Oxford University Press, 1983, pp. 3-63.

Plewig, G., Scheuber, E., Reuter, B., and Waidelich, W.: Thickness of corneocytes. In Marks, R. and Plewig, G. (eds): "Stratum Corneum." New York: Springer-Verlag, 1983, pp. 171-174.

Scheuplein, R. J.: Mechanism of percutaneous absorption I: Routes of penetration and the influence of solubility, J. Invest. Dermatol. 45: 334-346, 1965.

Scheuplein, R. J.: Mechanism of percutaneous absorption II: Transient diffusion and the relative importance of various routes of skin penetration, J. Invest. Dermatol. 48: 79-88, 1967.

Scheuplein, R. J.: Percutaneous absorption after twenty-five years: Or "old wine in new wineskins." J. Invest. Dermatol. 67: 31-38, 1976.

Scheuplein, R.: Skin as a barrier. In Jarrett, A. (ed): “The Physiology and Pathophysiology of Skin (Vol. 5). New York: Academic Press, 1978, pp. 1693-1730.

Scheuplein, R. J. and Bronaugh, R. L.: Percutaneous absorption. In Goldsmith, L. A. (ed): "Biochemistry and Physiology of the Skin (Vol. II) New York: Oxford University Press, 1983, pp. 1255-1295.

Shaw, J. E. and Chandrasekaran, R: Controlled topical delivery of drugs for systemic action, Drug Metab. Rev. 8: 223-233, 1978.

Steinert, P. M.: Epidermal keratin: Filaments and matrix. In Marks, R. and Plewig, G. (eds): "'Stratum Coreneum." New York: Springer-Verlag, 1983, pp. 25-38.

Walters, K. A.: Percutaneous absorption and transdermal delivery, Pharm. Technol. March 1986: 30, $32,34,36,40$, and $42,1986$.

Wester, R. C.: In vivo skin and nitroglycerine transdermal delivery, In Bronaugh, R. L. and Maibach, H. I. (eds): "Percutaneous Absorption." New York: Marcel Dekker, Inc., 1985, pp. 541-546.

Windholz, M. (ed): "The Merck Index," 10th edition. Rahway, NJ: Merck and Company, Inc., 1983.

Yalkowsky, S. H.: Solubility and solubilization of nonelectrolytes. In Yalkowski, S. H. (ed): "Techniques of Solubilization of Drugs." New York: Marcel Dekker, 1981, pp. 1-14. 